body of a small young shark. It was about eighteen inches long. I secured this as evidence. This locality is about 170 miles from the mouth of the river.

During the six months we were in the country, the bed of the river, which varies from 50 to 800 yards in width, was almost dry, with the exception of deep pools at intervals connected with each other by a narrow stream, often very shallow, running under the high banks. In the summer time the river is deeply flooded, the water rising ten to twenty feet (as shown by drift wood in trees) above the banks, in many places from forty to fifty feet high. The force of the flood might at its height prevent fish going up, but they could easily ascend in the intermediate season. In some cases the fish must have lived months in the upper waters, for portions of the Margaret, at least, are absolutely dry in the winter season, May to November usually.

I am not aware that such a circumstance has ever been noted before. If not, the fact is sufficientiy interesting in itself. It is also important from a geological point of view, as showing that some caution must be observed in the classification of strata as freshwater or marine on the evidence of fish alone. No doubt many of these remains are embedded in the river detritus, and if discovered at some future time when the physical geology of the country has altered, might lead to the conclusion that these deposits were of marine origin.

EDWARD F. HARDMAN,

H.M. Geological Survey, Government Geologist

Perth, Western Australia, January 28

\section{The Zodiacal Light}

ONE of the members of the staff of this establishment, Mr. E. G. Constable, ob̀served a brilliant appearance of the zodiacal light at about $7 \mathrm{p} . \mathrm{m}$. on the evening of Wednesday the 5 th inst., the cone of light being exceedingly well defined. The phenomenon was not visible long, having completely disappeared by $7.20 \mathrm{p} . \mathrm{m}$.

Kew Observatory, Richmond, Surrey, March 7

\section{THE AXIOMS OF GEOMETRY}

SIN

$\mathrm{NCE}$ the time when Riemann and Helmholtz began their investigations on the axioms of geometry so much has been written on this subject in learned papers and in a more or less popular form that it might have appeared superfluous again to call the attention of writers on, and teachers of, elementary geometry to it, had it not been for the publication a year or two ago of a new edition of the first six books of Euclid's "Elements," with annotations and notes, by Prof. Casey. I hope the eminent author of this in many respects excellent book will excuse me for criticising some points in it, and making them the opportunity for again returning to the question about the axioms in geometry.

The points I object to besides his treatment of Book V., of which I may possibly say a few words on another occasion, is contained in Note B at the end of the book. Here Prof. Casey gives Legendre's and Hamilton's proofs of I. 32, that the sum of the interior angles of any triangle is equal to two right angles, implying, of course, that he considers these proofs valid, proofs which are independent of the theory of parallels. The theorem in question depends in Euclid upon Axiom XII., and all depends upon the question whether this axiom is necessary. For the two propositions in this axiom and in Theorem I. 32 stand in such a relation that either is a consequence of the other. Hence if I. 32 can be proved independently, the Axiom XII. changes into a theorem. But the investigations above referred to show that it is this axiom which tells us what kind of a surface the plane really is, and that until this axiom is introduced all propositions apply equally well to the spherical and to the plane surface.

I select for discussion the "quaternion proof" given by Sir William Hamilton, this being the easiest of the two. But that by Legendre can be treated in exactly the same way.

Hamilton's proof consists in the following :--
One side $\mathrm{A} B$ of the triangle $\mathrm{A} \mathrm{B} \mathrm{C}$ is turned about the point $B$ till it lies in the continuation of $B C$; next, the line $\mathrm{B} C$ is made to slide along $\mathrm{B} C$ till $\mathrm{B}$ comes to $\mathrm{C}$, and is then turned about $\mathrm{C}$ till it comes to lie in the continuation of A C. It is now again made to slide along $\mathrm{C} \mathrm{A}$ till the point $B$ comes to $A$, and is turned about $A$ till it lies in the line A B. Hence it follows, since rotation is independent of translation, that the line has performed a whole revolution, that is, it has been turned tbrough four right angles. But it has also described in succession the three exterior angles of the triangle, hence these are together equal to four right angles, and from this follows at once that the interior angles are equal to two right angles.

To show how erroneous this reasoning is-in spite of Sir William Hamilton and in spite of quaternions-I need only point out that it holds exactly in the same manner for a triangle on the surface of the sphere, from which it would follow that the sum of the angles in a spherical triangle equals two right angles, whilst this sum is known to be always greater than two right angles. The proof depends only on the fact, that any line can be made to coincide with any other line, that two lines do so coincide when they have two points in common, and further, that a line may be turned about any point in it without leaving the surface. But if instead of the plane we take a spherical surface, and instead of a line a great circle on the sphere, all these conditions are again satisfied.

The reasoning employed must therefore be fallacious, and the error lies in the words printed in italics; for these words contain an assumption which has not been proved. In fact they contain an axiom which completely replaces Euclid's Axiom XII., viz. it expresses that property of a plane which differentiates it from the sphere.

On the sphere it is, of course, not true that rotation is independent of translation, simply because every translation-sliding along a great circle-is a rotation about the poles of the great circle.

From this it might be said to follow that the calculus of quaternions must be wrong. But this again is not correct. The fact is that the celebrated author of this calculus had built it up with the full knowledge of the fundamental space properties in his mind, and making full use of them. Afterwards, on reasoning backwards, he got these space properties out of his formulæ, forgetting that they were exactly the facts with which he started. The process is, as far as logic is concerned, not very different from that practised by some alchemists, who pretended to make gold, and actually did produce gold out of their crucibles, but only as much as they had themselves put in.

The following considerations may help to clear up this point still further :-

Prof. Sylvester once conceived, in illustration of some points connected with our subject, an infinitely thin bookworm living in a surface, and consequently limited in its space conceptions to the geometry on such surface. In a similar manner we may imagine an intelligent being consisting merely of an eye occupying a fixed point in space, but capable of perceiving rays of light in every direction. For such a being space would have two dimensions only, but in this space it could conceive figures for which most of Euclid's definitions and all axioms with the exception of the twelfth, and therefore all propositions up to the twenty-sixth in the first book, would hold. Only the names point, line, angle, \&c., would stand for objects different to those which they represent to our mind. Nothing can put the vagueness of Euclid's definitions and the real nature of his axioms, viz. that they contain the real logical definitions of the geometrical entities, in a clearer light than the fact that it is possible to use these so-called definitions for objects quite different from those to which Euclid applied them.

To return to our imaginary being : let us suppose it capable of studying Euclid. A ray of ligbt, that is, a line, 